\title{
Structures of Usutu SAAR-1776 virus: a comparison with known structures of mature flaviviruses \\ Baldeep Khare ${ }^{1}$, Thomas Klose ${ }^{2}$, Qianglin Fang ${ }^{3}$, Michael Rossmann ${ }^{4}$, Richard Kuhn ${ }^{5}$ \\ ${ }^{1}$ Purdue University ${ }^{2}$ Purdue University, ${ }^{3}$ Purdue University, ${ }^{4}$ Purdue University, ${ }^{5}$ Purdue University bkhare@purdue.edu
}

Flaviviruses are arthropod-borne viruses that are most commonly transmitted to humans via mosquitoes or ticks. These viruses are notorious for their infrequent, sudden outbreaks in the human population, which involve severe disease manifestations such as encephalitis or hemorrhagic fever; yet, the details of immunopathogenesis of the viruses and their interplay are not fully understood. Usutu virus (USUV), a flavivirus belonging to the Japanese encephalitis serocomplex and serologically closely related to the West Nile virus, is rampant in Europe and increasingly being recognized for its ability to cause severe neurological complications in humans. However, the prototype African strain, Usutu SAAR-1776 virus, is comparatively attenuated. To understand viral pathogenesis and combat infection effectively, we sought to determine the structure of USUV SAAR-1776. Since the first structure of mature Dengue determined in 2002 using single-particle electron microscopy, higher resolution studies of flaviviruses were till recently limited to the structure of the Zika virus at $3.1 \AA$ resolution. Here, we describe the structures of mature USUV, solved using single-particle cryo-electron microscopy to a resolution of $2.4 \AA$, the highest resolution for a mature flavivirus particle to-date, thus providing unprecedented detail of the structural proteins forming the icosahedral particle. We compare the USUV structures with previously determined structures of mature flaviviruses and highlight 1) the commonalities of a basic mature flavivirus particle, 2) features observed as a direct result of the high resolution of USUV structures and that may have implications for the wider field of flavivirus biology, and 3) unique features of the USUV particle that provides new knowledge for USUV pathogenesis. 